\title{
A DOUBLE STIRLING FORMULA
}

\author{
J. R. QUINE AND RICHARD R. SONG
}

(Communicated by Dennis A. Hejhal)

\begin{abstract}
We give an asymptotic expansion as $M \rightarrow \infty, N \rightarrow \infty$ of $\Pi(m+n \tau)^{2}$, where the product is over $1 \leq|m| \leq M, 1 \leq|n| \leq N$, $(m, n) \neq(0,0)$. The formula is analogous to the classical Stirling expansion on $M$ !. Of special interest is the constant term in the expansion, which involves the Dedekind eta function $\eta(z)$. Finding this constant is related to the Kronecker limit formula for the derivative at 0 of the zeta function $Z(s)=\sum_{m, n}^{\prime}|m+n \tau|^{-2 s}$. We consider instead the zeta function without absolute values.
\end{abstract}

\section{INTRODUCTION}

Consider the classical Stirling expansion,

$$
\log N !=\left(N+\frac{1}{2}\right) \log N-N+\log \sqrt{2 \pi}+O\left(N^{-1}\right)
$$

as $N \rightarrow \infty$. The terms going to infinity in the expansion can be obtained by the Euler summation formula, but the constant term $\log \sqrt{2 \pi}$ must be evaluated by a different technique (see, e.g., [Bo, p. 253]). Now consider the Riemann zeta function $\zeta(s)=\sum_{n=1}^{\infty} n^{-s}$ for $\operatorname{Re} s>1$, which can be extended by methods of analytic continuation to an entire meromorphic function which is analytic at $s=0$. The derivative at zero is

$$
\zeta^{\prime}(0)=-\log \sqrt{2 \pi},
$$

precisely the negative of the constant in Stirling's expansion. In fact, the value of $\zeta^{\prime}(0)$ can be used to obtain Stirling's constant. We outline how this is done in $\S 2$.

Now suppose we have a zeta function corresponding to a two-dimensional lattice, say $Z(s)=\sum^{\prime}|m+n \tau|^{-2 s}$, with $\operatorname{Im} \tau>0$, where the prime indicates the sum over all integer pairs $(m, n) \neq(0,0)$. The evaluation of $Z^{\prime}(0)$ is known as Kronecker's limit formula. It says that

$$
Z^{\prime}(0)=-\log (2 \pi)^{2}|\eta(\tau)|^{4},
$$

where $\eta(\tau)$ is the Dedekind eta function, $e^{\pi i \tau / 12} \prod_{k=1}^{\infty}\left(1-e^{2 \pi i k \tau}\right)$.

Received by the editors February 15, 1992.

1991 Mathematics Subject Classification. Primary 11F20, 11M41; Secondary 11E45.

Key words and phrases. Stirling expansion, Dedekind eta function, Kronecker limit formula, zeta regularized product. 
The question arises whether a "Stirling" formula exists for equation (3), just as equation (1) corresponds to equation (2). This would be an asymptotic formula for $\prod_{|m| \leq M,|n| \leq N}^{\prime}|m+n \tau|^{2}$ as $M$ and $N$ go to $\infty$. For the case $\tau=i$ this gives a natural generalization of $(M !)^{4}=\prod_{|m| \leq M}^{\prime} m^{2}$. The purpose of this paper is to give such a formula (see $\S 4$, Theorem), which we call the double Stirling formula. The corollary deals with the special case when $\tau=i$.

We found that it was necessary to derive the formula for the product without absolute values, and in doing so we needed to consider the zeta function $Z_{1}(s)=$ $\sum^{\prime}(m+n \tau)^{-s}$ for a suitable choice of $\log (m+n \tau)$. The value $Z_{1}^{\prime}(0)$ gave us the constant in the expansion. The Kronecker limit formula did not directly apply.

We prefer to consider the evaluation of the derivatives of zeta functions at 0 in the context of zeta regularized products, which we describe in $\S 2$. In $\S 3$ we give motivation for the proof by considering how to prove the classical Stirling formula starting with (2). In $\S 4$ we state the theorem and give the proof. The idea for the proof comes from two main sources. The first is Barnes's proof for his asymptotic expansion of multiple gamma functions [Ba]. The second is Shintani's treatment [S] of the Kronecker limit formula using the double gamma function. For a synthesis of these ideas and others, see our paper [QHS]. It was in this context that the problem considered here arose.

\section{ZETA REGULARIZED PRODUCTS}

Let $\lambda_{k}, k=1,2, \ldots$, be a sequence of nonzero complex numbers, and let $Z(s)=\sum_{k=1}^{\infty} \lambda_{k}^{-s}$ be the zeta function associated to this sequence. The zeta function will depend on the particular choice of $\arg \lambda_{k}$, and for a real sequence the arg is chosen to be 0 . We will assume that the series for $Z(s)$ converges absolutely in some half-plane $\operatorname{Re} s>\alpha$ and that it can be analytically continued to a meromorphic function in the plane which is holomorphic at $s=0$. This will be the case for all the examples that follow. We define the zeta regularized product of the sequence by

$$
\prod_{z} \lambda_{k}=\exp \left(-Z^{\prime}(0)\right)
$$

Some properties of the zeta regularized product are easily established from the definition. First, if $a$ is any nonzero number, then

$$
\prod_{z} a \lambda_{k}=a^{Z(0)} \prod_{z} \lambda_{k}
$$

provided the arguments are chosen so that $\arg a \lambda_{k}=\arg a+\arg \lambda_{k}$. We will call the sequence $a \lambda_{k}$ the rotated sequence. Next, if $\lambda_{k}$ is the union of two sequences $\lambda_{k}^{1}$ and $\lambda_{k}^{2}$ renumbered in any way, then

$$
\prod_{z} \lambda_{k}=\prod_{z} \lambda_{k}^{1} \prod_{z} \lambda_{k}^{2}
$$

Choosing $\log \lambda_{k}^{2}=2 \log \lambda_{k}$ and $\log \overline{\lambda_{k}}=\overline{\log \lambda_{k}}$, we also have

$$
\begin{aligned}
\prod_{z} \lambda_{k}^{2} & =\left(\prod_{z} \lambda_{k}\right)^{2}, \\
\prod_{z} \overline{\lambda_{k}} & =\overline{\left(\prod_{z} \lambda_{k}\right)} .
\end{aligned}
$$


We will also be concerned with zeta regularized products of the form $\Pi_{z}\left(\lambda_{k}+z\right)$. The convention on these is that $\arg \left(\lambda_{k}+z\right)$ is approximately equal to $\arg \lambda_{k}$ for $\left|\lambda_{k}\right|$ large. This is sufficient to determine the product, since changing a finite number of arguments in the product does not change the product. In the product $\Pi_{z}\left(\lambda_{k}+z\right)$ we can allow a finite number of the $\lambda_{k}$ 's to be zero by considering the contribution of those factors as $z^{l}$, where $l$ is the total number of $\lambda_{k}$ 's which are zero.

More information on zeta regularized products and methods for computing them can be found in [QHS]. Here is a list of some of the products mentioned there and the value of the corresponding zeta function $Z$ at 0 . We assume that $\operatorname{Im} \tau>0$ and $-\pi \leq \arg (m+n \tau)<\pi$ for all integers $m$ and $n$. Also we write $P(\tau)=\prod_{n=1}^{\infty}\left(1-e^{2 \pi i n \tau}\right)$ and $\eta(\tau)=e^{\pi i \tau / 12} P(\tau)$ for the Dedekind eta function. The symbol $\Pi^{\prime}$ denotes the product for $(m, n) \neq(0,0)$.

$$
\begin{gathered}
\prod_{m \geq 1}(m+z)=\frac{\sqrt{2 \pi}}{\Gamma(z+1)}, \quad Z(0)=\frac{1}{2}+z ; \\
\prod_{z}^{m}(m+\tau)=1-e^{2 \pi i \tau}, \quad Z(0)=0 ; \\
\prod_{\substack{n \geq 1 \\
m \geq 0}}(m+n \tau)=P(\tau), \quad Z(0)=0 ; \\
\prod_{m, n}^{\prime}(m+n \tau)=2 \pi i[P(\tau)]^{2}, \quad Z(0)=1 ; \\
\prod_{m, n}^{\prime}|m+n \tau|=2 \pi|\eta(\tau)|^{2},
\end{gathered}
$$

We note that (10) appears to follow from (9) by taking an iterated product, but this procedure must be justified. We also note from property (4) that products (9) and (10) do not change if the sequence is rotated since $Z(0)=0$. Also we remark that

$$
\left|\prod_{z}(m+n \tau)\right| \neq \prod_{z}|m+n \tau|,
$$

so (11) does not directly give Kronecker's limit formula (12).

\section{STIRLING'S FORMULA AND ZETA REGULARIZED PRODUCTS}

To motivate $\S 4$, we give a brief discussion of the classical Stirling formula from the point of view of zeta regularized products. Formula (1) can also be written as $N !=N^{N+1 / 2} e^{-N} \sqrt{2 \pi} e^{\varepsilon}$, where $\varepsilon=O\left(N^{-1}\right)$ as $N \rightarrow \infty$. We note that $\sqrt{2 \pi}$ in the formula is $\prod_{z n=1}^{\infty} n$. The following analysis shows why this is so. A similar analysis will be used in the proof of the double Stirling formula.

Let

$$
\zeta(s, x)=\sum_{n=1}^{\infty}(n+x)^{-s}
$$

for $x>0$ and $\operatorname{Re} s>1$, so $\zeta(s, 0)=\zeta(s)$ is the Riemann zeta function. Use the Mellin transform formula [SG, p. 364] to get

$$
\zeta(s, x)=\frac{1}{\Gamma(s)} \int_{0}^{\infty} e^{-x t} \theta(t) t^{s-1} d t
$$


where $\theta(t)=\sum_{n=1}^{\infty} e^{-n t}=\left(e^{t}-1\right)^{-1}$. The expansion $\theta(t)=t^{-1}-\frac{1}{2}+O(t)$ as $t \rightarrow 0^{+}$can be integrated to give for $s>-1$ the expansion

$$
\zeta(s, x)=\frac{1}{s-1} x^{1-s}-\frac{1}{2} x^{-s}+s O\left(x^{-1-s}\right)
$$

as $x \rightarrow \infty$ [SG, p. 458]. This expansion can also be differentiated at $s=0$ to give

$$
\left.\frac{d}{d s} \zeta(s, x)\right|_{s=0}=-\log \prod_{n=1}^{\infty}(n+x)=\left(x+\frac{1}{2}\right) \log x-x+O\left(x^{-1}\right)
$$

as $x \rightarrow \infty$. Now writing (using (5))

$$
N !=\prod_{n=1}^{\infty} n / \prod_{n=1}^{\infty}(n+N)=\sqrt{2 \pi} / \prod_{n=1}^{\infty}(n+N)
$$

gives Stirling's formula.

\section{The double Stirling formula}

Theorem (double Stirling formula). Suppose $\operatorname{Im} \tau>0, M$ and $N$ are positive integers, and $\arg ((M+N \tau) /(M-N \tau))$ and $\arg (-(M-N \tau) /(M+N \tau))$ are chosen in the interval $(0, \pi)$. Then

$$
\prod_{\substack{|m| \leq M \\|n| \leq N \\(m, n) \neq(0,0)}}(m+n \tau)^{2}=\exp (-4(3 M N+M+N))\left(M^{2}-N^{2} \tau^{2}\right)^{\gamma}\left(-\frac{M-N \tau}{M+N \tau}\right)^{\alpha}
$$

$$
\cdot\left(\frac{M+N \tau}{M-N \tau}\right)^{\beta}(2 \pi)^{2}(n(\tau))^{4} \exp (\varepsilon)
$$

where $\gamma=(2 M+1)(2 N+1), \alpha=-\tau\left(\frac{1}{3}+2 N(N+1)\right), \beta=\frac{1}{\tau}\left(\frac{1}{3}+2 M(M+1)\right)$, $\eta$ is the Dedekind eta function, and $\varepsilon=O\left(M^{-1}\right)+O\left(N^{-1}\right)$ as $M \rightarrow \infty$ and $N \rightarrow \infty$.

Corollary. With notation as in the theorem,

$$
\begin{aligned}
M ! N ! & \prod_{\substack{1 \leq m \leq M \\
1 \leq n \leq N}}\left(m^{2}+n^{2}\right)=\left(M^{2}+N^{2}\right)^{(M+1 / 2)(N+1 / 2)} \\
& \cdot \exp \left(-3 M N-M-N+M(M+1) \arctan \frac{N}{M}+N(N+1) \arctan \frac{M}{N}\right) \\
& \cdot \sqrt{2 \pi} \prod_{n=1}^{\infty}\left(1-e^{-2 n \pi}\right) \exp (\varepsilon) .
\end{aligned}
$$

We note that since $\prod_{n=1}^{\infty}\left(1-e^{-2 \pi n}\right)=0.99812 \ldots$ is very close to 1 , the constant in the corollary is very nearly $\sqrt{2 \pi}$, the constant in the classical Stirling expansion. 
To prove the theorem, let $a$ and $b$ be complex numbers with positive real parts. For $\operatorname{Re} s>2, t>0$, let

$$
\begin{aligned}
Z(s, a, b, z) & =\sum_{m, n=1}^{\infty}(m a+n b+z)^{-s}, \\
\theta(t) & =\sum_{m, n=1}^{\infty} e^{-(m a+n b) t},
\end{aligned}
$$

where the principal branch of $\log$ is used to define $Z$. For $t>0$ we find the expansion

$$
\theta(t)=\left(e^{a t}-1\right)^{-1}\left(e^{b t}-1\right)^{-1}=c_{2} t^{-2}+c_{1} t^{-1}+c_{0}+O(t)
$$

as $t \rightarrow 0^{+}$, where

$$
\begin{aligned}
& c_{2}=c_{2}(a, b)=\frac{1}{a b}, \\
& c_{1}=c_{1}(a, b)=-\frac{1}{2}\left(\frac{1}{a}+\frac{1}{b}\right), \\
& c_{0}=c_{0}(a, b)=\frac{1}{4}+\frac{1}{12}\left(\frac{a}{b}+\frac{b}{a}\right) .
\end{aligned}
$$

Now we write $Z$ as a Laplace type integral

$$
Z(s, a, b, z)=\frac{1}{\Gamma(s)} \int_{0}^{\infty} \theta(t) t^{s-1} e^{-z t} d t
$$

for $\operatorname{Re} s>2$. Using (15) to integrate the expansion (13) [SG, p. 458], we get, for $s>-1, \operatorname{Re} z>0$,

$$
Z(s, a, b, z)=\frac{1}{(s-1)(s-2)} c_{2} z^{2-s}+\frac{1}{s-1} c_{1} z^{1-s}+c_{0} z^{-s}+s O\left(|z|^{-1-s}\right)
$$

as $z \rightarrow \infty,|\arg | \leq \frac{\pi}{2}-\delta, \delta>0$.

We note from (14) that if $s=0$, the expansion (16) is invariant if $a, b$, and $z$ are replaced by $\sigma a, \sigma b$, and $\sigma z$, respectively, for $\sigma$ any nonzero complex number. Thus we can remove the restriction on the real parts of $a$ and $b$, and the expansion holds as $z \rightarrow \infty$ in any closed sector of angle $<\pi$ contained in some open half-plane through the origin containing $a$ and $b$. The branch of $\log$ must be chosen appropriately in defining $Z$; i.e., $\log (\sigma(m a+n b+z))=$ $\log \sigma+\log (m a+n b+z)$. This remark holds also for the following differentiated version at $s=0$.

Now the expansion (16) may be differentiated at $s=0$ [SG, p. 462], and we get, for $z \rightarrow \infty$,

$$
Z^{\prime}(0, a, b, z)=A(a, b, z)+O\left(|z|^{-1}\right),
$$

where

$$
\begin{aligned}
A(a, b, z) & =\left.\frac{d}{d s}\left(\frac{1}{(s-1)(s-2)} c_{2} z^{2-s}+\frac{1}{s-1} c_{1} z^{1-s}+c_{0} z^{-s}\right)\right|_{s=0} \\
& =\frac{c_{2} z^{2}}{z}\left(-\log z+\frac{3}{2}\right)+c_{1} z(\log z-1)-c_{0} \log z
\end{aligned}
$$


We can deduce from the definition of $Z$ (or from (5)) that

$$
\begin{aligned}
\sum_{\substack{1 \leq m \leq M \\
1 \leq n \leq N}} \log (m a+n b)= & -Z^{\prime}(0, a, b, 0)+Z^{\prime}(0, a, b, M a) \\
& +Z^{\prime}(0, a, b, N b)-Z^{\prime}(0, a, b, M a+N b) .
\end{aligned}
$$

Hence by (17)

$$
\sum_{\substack{1 \leq m \leq M \\ 1 \leq n \leq N}} \log (m a+n b)=B(a, b, M, N)+Z^{\prime}(0, a, b, 0)+\varepsilon,
$$

where

$$
B(a, b, M, N)=A(a, b, M a)+A(a, b, N b)-A(a, b, M a+N b)
$$

and $\varepsilon=O\left(M^{-1}\right)+O\left(N^{-1}\right)$. Thus letting $a=1, b=\tau$ and $a=-1, b=\tau$ in (18) and adding the results, we have

$$
\begin{gathered}
\sum_{\substack{|m| \leq M \\
1 \leq n \leq N}} \log (m+n \tau)=\sum_{\substack{1 \leq|m| \leq M \\
1 \leq n \leq N}} \log (m+n \tau)+\sum_{1 \leq n \leq N} \log (n \tau) \\
=\left\{B(1, \tau, M, N)+B(-1, \tau, M, N)+\left(N+\frac{1}{2}\right) \log N \tau-N\right\} \\
\quad+\left\{Z^{\prime}(0,1, \tau, 0)+Z^{\prime}(0,-1, \tau, 0)+\log \left(\sqrt{2 \pi} \tau^{-1 / 2}\right)\right\}+\varepsilon
\end{gathered}
$$

where we used Stirling's formula (1). We compute the first term in brackets as

$$
\begin{aligned}
-3 M N-N+\pi i\left(-\frac{1}{4}+\frac{M}{2}+\beta+\frac{\tau}{12}\right)+\left(\frac{1}{2}+M\right) \log M \\
+\frac{1}{4}(\gamma+\alpha-\beta) \log (-M+N \tau)+\frac{1}{4}(\gamma+\beta-\alpha) \log (M+N \tau),
\end{aligned}
$$

where $\gamma, \alpha$, and $\beta$ are as stated in the theorem. We recognize the second term in brackets as the zeta regularized product $(10), P(\tau)$.

Now adding another Stirling expansion (1) to (19) and exponentiating yields the theorem. The corollary follows easily by substituting $\tau=i$ and using the identities

$$
\begin{gathered}
\arg \left(\frac{M+N i}{M-N i}\right)=2 \arctan \frac{N}{M}, \\
\arg \left(-\frac{M-N i}{M+N i}\right)=2 \arctan \frac{M}{N}, \\
\arctan \left(\frac{M}{N}\right)+\arctan \left(\frac{N}{M}\right)=\pi
\end{gathered}
$$

\section{REFERENCES}

[Ba] E. W. Barnes, The theory of the double gamma function, Philosophical Transactions of the Royal Society (A) 196 (1901), 265-388.

[Bo] R. P. Boas, Invitation to complex analysis, Random House/Birkhäuser, New York, 1987.

[QHS] J. R. Quine, S. H. Heydari, and R. Y. Song, Zeta regularized products, Trans. Amer. Math. Soc. (to appear). 
[SG] G. Sansone and J. Gerretsen, Lectures on the theory of functions of a complex variable, Noordhoff, Groningen, 1960.

[S1] T. Shintani, A proof of the classical Kronecker limit formula, Tokyo J. Math. 3 (1980), 191-199.

Department of Mathematics, Florida State University, Tallahassee, Florida 323063027

E-mail address, J. R. Quine: quine@math.fsu.edu

E-mail address, R. R. Song: song@math.fsu.edu 